\title{
Vibration control of an elastic strip by a singular force
}

\author{
EROL UZAL and BANU KORBAHTI \\ Faculty of Engineering, Department of Mechanical Engineering, Istanbul \\ University, Istanbul 34320, Turkey \\ e-mail: korbahti@istanbul.edu.tr
}

MS received 10 September 2008; revised 27 August 2009; accepted 17 December 2009

\begin{abstract}
Vibration characteristics of an elastic plate in the shape of an infinite strip are changed by applying a lateral concentrated force to the plate. The homogeneous, isotropic, elastic plate is infinite in the $x$-direction and the sides are simply supported. The size of the force is changed in proportion to the displacement measured at a certain point of the plate. The proportionality constant serves as the control parameter. The mathematical formulation of this distributed control problem and its analytical solution in terms of the vibration frequencies of the plate are given. The vibration frequencies are plotted as a function of the control parameter.
\end{abstract}

Keywords. Control; vibration; distributed parameter system; plate.

\section{Introduction}

Predicting resonance frequency of plates is an important technological and scientific problem for which many fairly standard methods have been developed. Recently, controlling structural vibrations by various passive and active means is being vigorously investigated. Piezoelectric sensors and actuators have been chosen in most of the current studies, for example, Chandiramani et al (2004); Han \& Lee (1999); Sadri et al (1999); Shete et al (2007), although there are other alternatives. Obviously, being able to control plate vibrations would have profound results especially in aerospace structures. Applying control theory to simpler models which use ordinary differential equations has been considered in a number of studies. The approximate ordinary differential equations have been obtained by finite element or RayleighRitz methods in a number of studies pertaining to control of flutter in aerospace structures and other vibration problems (Moon \& Kim 2005; Frampton et al 1996; Döngi et al 1996; Forster \& Yang 1998; Han et al 2006; Kwak \& Heo 2007; Walker \& Yaneske 1976; Bingham et al 2001; Young et al 2002; De Matteis et al 2008; Bos \& Casagrande 2003; Luo et al 2008; Bolotin et al 2001; Shen \& Homaifar 2001; Luo et al 2008; Ro \& Baz 2002; Hurlebaus et al 2008) using piezoelectric actuators. (Benjeddou 2000; Sunar \& Rao 1999) are surveys of piezoelectric control of flexible structures. (Yang \& Ngoi 2000; Gaudenzi et al 1997; Cai \& Yang 2006) use piezoelectric actuators in controlling vibration and shape of beams. Varadarajan et al (1998) applied piezoelectric materials in the shape control of composite plates. De Fonseca et al 1999; Halim et al (2008) studied to find optimal sensor and actuator locations in 


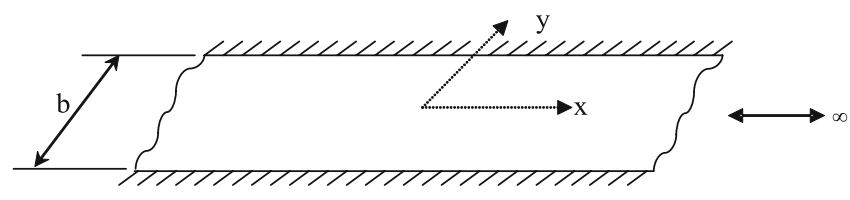

Figure 1. Problem geometry.

control of flexible structures. Chow \& Maestrello (1997) considered control of nonlinear plate vibrations by an applied pressure, but the pressure is assumed to be present at every point on the surface of the plate. In the present paper, an elastic plate in the shape of an infinite strip is considered; but we assume that the measurement and actuation are at single points rather than distributed over the whole plate. Since this is a distributed parameter system from the control theory point of view, the standardised control techniques can not be used even for the linear problem. One approach has been discretizing the problem so that the resulting lumped parameter model is a good representation of the original structure. Then the standard methods of control can be applied. In this paper, we tried to solve the distributed parameter system directly.

\section{Problem formulation and solution}

The homogeneous, isotropic, elastic plate is infinite in the $x$-direction and has width $b$ in $y$ direction (figure 1). The sides are simply supported. The eigenfrequencies in this case can be easily computed assuming plane waves propagating in the $x$-direction. Our purpose is to modify the eigenfrequencies by feedback control. Piezoelectric actuators are widely used for controlling vibrating systems; here, we propose a simpler approach. We assume that the deformation is measured at a point on the plate at all times and this information is then used to apply a concentrated force to the plate at another point. The magnitude of the force is chosen to be proportional to the measured displacement; but the solution to be presented below is sufficiently general so that it can easily be extended to forces proportional to velocity or acceleration or their linear combination. This control scheme can be applied by measuring the displacement directly by a laser range finder (or perhaps measuring the acceleration and obtaining the displacement) and applying the required control force by an electrodynamic shaker or similar linear actuator.

Controlled plate vibrations are governed by

$$
D\left(\frac{\partial^{4} w}{\partial x^{4}}+2 \frac{\partial^{4} w}{\partial x^{2} \partial y^{2}}+\frac{\partial^{4} w}{\partial y^{4}}\right)+\rho_{p} h_{p} \frac{\partial^{2} w}{\partial t^{2}}+Q w\left(x_{1}, 0, t\right) \delta\left(x-x_{2}\right) \delta(y)=0,
$$

$w(x, y, t)$ shows the plate deflection, $\rho_{p}$ and $h_{p}$ are plate's density and thickness, respectively, and $D=E h_{p}^{3} / 12\left(1-v^{2}\right)$ is the bending rigidity of the plate. The last term in Eq. (1) models the control action in which $w\left(x_{1}, 0, t\right)$ shows the plate deflection measured on the $y$-axis at the location $x=x_{1}$ at all times. The factors including the delta distributions $\delta\left(x-x_{2}\right) \delta(y)$ shows that control force is applied on the $x$-axis at the location $x=x_{2}$ and $Q$ is the proportionality constant (dimension $\mathrm{N} / \mathrm{m}$ ) which is the design parameter from the control theory point of view. We choose the measurement and actuation on the $x$-axis but they can be at any point on the plate. The dynamics of the means by which the measurements are made and the control force is applied was neglected. Because of the form of the last term, this is also an 
interesting mathematical problem. The plate is assumed to be simply supported along the side walls, i.e.

$$
w=\frac{\partial^{2} w}{\partial y^{2}}=0 \text { at } y= \pm b / 2 .
$$

We use the following dimensionless quantities

$$
x^{*}=\frac{x}{b}, y^{*}=\frac{y}{b}, w^{*}=\frac{w}{b}, t^{*}=\frac{t}{\sqrt{\frac{\rho_{p} h_{p} b^{4}}{D}}} .
$$

Then equations (1) and (2) become, discarding the stars

$$
\begin{aligned}
& \frac{\partial^{4} w}{\partial x^{4}}+2 \frac{\partial^{4} w}{\partial x^{2} \partial y^{2}}+\frac{\partial^{4} w}{\partial y^{4}}+\frac{\partial^{2} w}{\partial t^{2}}+K w\left(x_{1}, 0, t\right) \delta\left(x-x_{2}\right) \delta(y)=0, \\
& w=\frac{\partial^{2} w}{\partial y^{2}}=0 \text { at } y= \pm 1 / 2
\end{aligned}
$$

where

$$
K=\frac{Q b^{2}}{D}
$$

is the non-dimensional control parameter.

In the absence of the control term, the solutions of equations (4) and (5) can be sought in the form of travelling waves in the $x$-direction. Due to the control term, we have to follow a different approach. Assuming plate deflections of the form

$$
w(x, y, t)=W(x, y) e^{i \omega t},
$$

equation (4) becomes

$$
\frac{\partial^{4} W}{\partial x^{4}}+2 \frac{\partial^{4} W}{\partial x^{2} \partial y^{2}}+\frac{\partial^{4} W}{\partial y^{4}}-\omega^{2} W+K W\left(x_{1}, 0\right) \delta\left(x-x_{2}\right) \delta(y)=0 .
$$

Taking Fourier transform of this equation with respect to $x$, we obtain

$$
\xi^{4} \hat{W}-2 \xi^{2} \frac{d^{2} \hat{W}}{d y^{2}}+\frac{d^{4} \hat{W}}{d y^{4}}-\omega^{2} \hat{W}+K W\left(x_{1}, 0\right) \delta(y) e^{i \xi x_{2}}=0 .
$$

Fourier transform pair with the following convention has been used

$$
\hat{f}(\xi)=\int_{-\infty}^{\infty} f(x) e^{i \xi x} d x, \quad f(x)=\frac{1}{2 \pi} \int_{-\infty}^{\infty} \hat{f}(\xi) e^{-i \xi x} d \xi .
$$

Boundary conditions become

$$
\hat{W}=\frac{d^{2} \hat{W}}{d y^{2}}=0 \text { at } y= \pm 1 / 2
$$


The method of variation of parameters finds the general solution from varying the parameters of the homogeneous solution. $C_{1}(y), C_{2}(y), C_{3}(y)$ and $C_{4}(y)$ are unknown functions of $y$ to be determined.

$$
\hat{W}=C_{1}(y) e^{r_{1} y}+C_{2}(y) e^{r_{2} y}+C_{3}(y) e^{r_{3} y}+C_{4}(y) e^{r_{4} y},
$$

where $r_{1}, r_{2}, r_{3}$ and $r_{4}$ are the roots of the algebraic equation

$$
r^{4}-2 \xi^{2} r^{2}+\left(\xi^{4}-\omega^{2}\right)=0
$$

and $C_{1}(y), C_{2}(y), C_{3}(y), C_{4}(y)$ satisfy

$$
\begin{aligned}
& C_{1}^{\prime} e^{r_{1} y}+C_{2}^{\prime} e^{r_{2} y}+C_{3}^{\prime} e^{r_{3} y}+C_{4}^{\prime} e^{r_{4} y}=0, \\
& r_{1} C_{1}^{\prime} e^{r_{1} y}+r_{2} C_{2}^{\prime} e^{r_{2} y}+r_{3} C_{3}^{\prime} e^{r_{3} y}+r_{4} C_{4}^{\prime} e^{r_{4} y}=0, \\
& r_{1}^{2} C_{1}^{\prime} e^{r_{1} y}+r_{2}^{2} C_{2}^{\prime} e^{r_{2} y}+r_{3}^{2} C_{3}^{\prime} e^{r_{3} y}+r_{4}^{2} C_{4}^{\prime} e^{r_{4} y}=0, \\
& r_{1}^{3} C_{1}^{\prime} e^{r_{1} y}+r_{2}^{3} C_{2}^{\prime} e^{r_{2} y}+r_{3}^{3} C_{3}^{\prime} e^{r_{3} y}+r_{4}^{3} C_{4}^{\prime} e^{r_{4} y}=-K W\left(x_{1}, 0\right) \delta(y) e^{i \xi x_{2}} .
\end{aligned}
$$

Prime denotes differentiation with respect to $y$. Equations (13) are solved by Cramer's rule to give

$$
\begin{aligned}
& C_{1}^{\prime}=D_{1}(y) K W\left(x_{1}, 0\right) \delta(y) e^{i \xi x_{2}} \\
& C_{2}^{\prime}=D_{2}(y) K W\left(x_{1}, 0\right) \delta(y) e^{i \xi x_{2}} \\
& C_{3}^{\prime}=D_{3}(y) K W\left(x_{1}, 0\right) \delta(y) e^{i \xi x_{2}} \\
& C_{4}^{\prime}=D_{4}(y) K W\left(x_{1}, 0\right) \delta(y) e^{i \xi x_{2}},
\end{aligned}
$$

where $D_{k}(y)$ are formed using the coefficients in equations (13), as the ratio of two determinants. Integrating equations (14) and substituting in equation (11),

$$
\begin{aligned}
\hat{W}= & E_{1} e^{r_{1} y}+E_{2} e^{r_{2} y}+E_{3} e^{r_{3} y}+E_{4} e^{r_{4} y} \\
& +\left[D_{1}(0) e^{r_{1} y}+D_{2}(0) e^{r_{2} y}+D_{3}(0) e^{r_{3} y}+D_{4}(0) e^{r_{4} y}\right] K W\left(x_{1}, 0\right) e^{i \xi x_{2}} h(y),
\end{aligned}
$$

$h(y)$ denotes the unit step function and $E_{k}$ are integration constants. Despite the appearance of the unit step function, $\hat{W}$ is continuous at $y=0$ because the square bracket in equation (15) becomes zero there. This can easily be seen by setting $y=0$ in equation (13a) and using equation (14). Note that equation (15) is not an explicit solution since the right-hand side includes $W\left(x_{1}, 0\right)$. Next, the solution, equation (15), is substituted in the boundary conditions equation (10) to determine the constants $E_{1}, E_{2}, E_{3}$ and $E_{4}$;

$$
\begin{aligned}
& E_{1} e^{-r_{1} / 2}+E_{2} e^{-r_{2} / 2}+E_{3} e^{-r_{3} / 2}+E_{4} e^{-r_{4} / 2}=0 \\
& r_{1}^{2} E_{1} e^{-r_{1} / 2}+r_{2}^{2} E_{2} e^{-r_{2} / 2}+r_{3}^{2} E_{3} e^{-r_{3} / 2}+r_{4}^{2} E_{4} e^{-r_{4} / 2}=0 \\
& E_{1} e^{r_{1} / 2}+E_{2} e^{r_{2} / 2}+E_{3} e^{r_{3} / 2}+E_{4} e^{r_{4} / 2} \\
& \quad+\left[D_{1}(0) e^{r_{1} / 2}+D_{2}(0) e^{r_{2} / 2}+D_{3}(0) e^{r_{3} / 2}+D_{4}(0) e^{r_{4} / 2}\right] K W\left(x_{1}, 0\right) e^{i \xi x_{2}}=0 \\
& r_{1}^{2} E_{1} e^{r_{1} / 2}+r_{2}^{2} E_{2} e^{r_{2} / 2}+r_{3}^{2} E_{3} e^{r_{3} / 2}+r_{4}^{2} E_{4} e^{r_{4} / 2} \\
& \quad+\left[r_{1}^{2} D_{1}(0) e^{r_{1} / 2}+r_{2}^{2} D_{2}(0) e^{r_{2} / 2}+r_{3}^{2} D_{3}(0) e^{r_{3} / 2}+r_{4}^{2} D_{4}(0) e^{r_{4} / 2}\right] K W\left(x_{1}, 0\right) e^{i \xi x_{2}}=0 .
\end{aligned}
$$



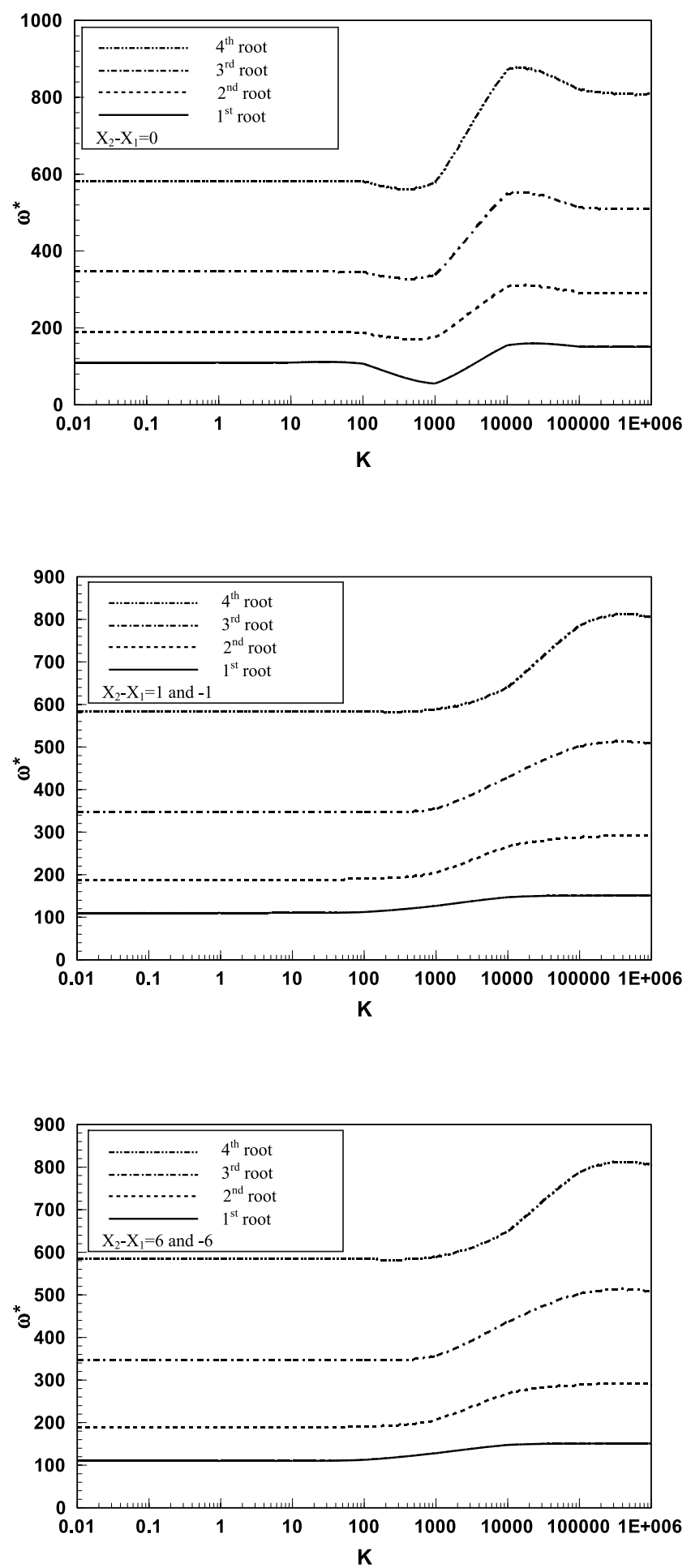

Figure 2. Change in the first four eigenvalues as function of the control parameter $K$ for $x_{2}-x_{1}=0$.

Figure 3. Change in the first four eigenvalues as function of the control parameter $K$ for $x_{2}-x_{1}=1$.

Figure 4. Change in the first four eigenvalues as function of the control parameter $K$ for $x_{2}-x_{1}=6$. 
Here, $E_{k}$ are again found by Cramer's rule and found like $E_{1}=H_{1} K W\left(x_{1}, 0\right) e^{i \xi x_{2}}, E_{2}=$ $H_{2} K W\left(x_{1}, 0\right) e^{i \xi x_{2}}, E_{3}=H_{3} K W\left(x_{1}, 0\right) e^{i \xi x_{2}} E_{4}=H_{4} K W\left(x_{1}, 0\right) e^{i \xi x_{2}}$, where $H_{k}$ are formed using the coefficients in equations (16), as the ratio of two determinants.

When the constants $E_{k}$ are substituted back into the solution, the result is

$$
\begin{aligned}
\hat{W}= & K W\left(x_{1}, 0\right) e^{i \xi x_{2}}\left\{\left[H_{1}+h(y) D_{1}(0)\right] e^{r_{1} y}+\left[H_{2}+h(y) D_{2}(0)\right] e^{r_{2} y}\right. \\
& \left.+\left[H_{3}+h(y) D_{3}(0)\right] e^{r_{3} y}+\left[H_{4}+h(y) D_{4}(0)\right] e^{r_{4} y}\right\} .
\end{aligned}
$$

We can simply write

$$
\hat{W}=K W\left(x_{1}, 0\right) e^{i \xi x_{2}} \hat{J}(\xi, y),
$$

where $\hat{J}(\xi, y)=e^{i \xi x_{2}}\left\{H_{1} e^{r_{1} y}+H_{2} e^{r_{2} y}+H_{3} e^{r_{3} y}+H_{4} e^{r_{4} y}\right\}$.

Taking inverse Fourier transform of equation (18), we obtain

$$
W(x, y)=K W\left(x_{1}, 0\right) J(x, y),
$$

where $J(x, y)$ is the inverse Fourier transform of the factors involving $\xi$ in equation (18). Writing $x=x_{1}$ and $y=0$ in equation (18), the terms $W\left(x_{1}, 0\right)$ cancel and we obtain

$$
1=J\left(x_{1}, 0\right) K
$$

as the eigenvalue equation. It involves, in addition to the eigenvalue $\omega$, control gain constant $K$, and measurement and actuation point locations on the $y$-axis, $x_{1}$ and $x_{2}$. Thus, we are able to retrieve the equation satisfied by the controlled eigenvalues although we did not solve the problem explicitly. Furthermore, written explicitly

$$
J\left(x_{1}, 0\right)=\frac{1}{2 \pi} \int_{-\infty}^{\infty} e^{i \xi\left(x_{2}-x_{1}\right)}\left(H_{1}+H_{2}+H_{3}+H_{4}\right) d \xi,
$$

depends only on the difference $x_{2}-x_{1}$ and not on $x_{1}$ and $x_{2}$ separately. This is also reasonable since the plate is infinite in the $x$-direction.

\section{Conclusions}

Equation (19) gives the eigenvalue $\omega$ as a function of the control parameter $K$ and the difference between measurement and actuation locations $x_{2}-x_{1}$. It was verified that equation (19) gives the eigenvalues without any control when $K$ approaches zero.

Figures 2, 3, 4 show the first four eigenvalues as a function of the control parameter $K$ for various values of $x_{2}-x_{1}$. It is to be noted that $H_{1}+H_{2}+H_{3}+H_{4}$ is an even function of $\xi$, therefore the results are independent of the sign $x_{2}-x_{1}$ which is also reasonable.

It appears that the (first four) eigenvalues do not change appreciably until the control parameter reaches the value of 100 . When measurement and actuation are at the same point $\left(x_{2}-x_{1}=0\right)$ the eigenvalues decrease slightly after $K=100$ and then they increase. For large values of $K$ the eigenvalues become independent of $K$ and each eigenvalue achieves a constant value regardless of the value of $x_{2}-x_{1}$. The control force required of the actuator is $Q w=\frac{K D}{b^{2}} w$, where $w$ is the measured displacement. The results presented show that the non-dimensional control constant should be larger than about 100 to have any effect on the plate eigenfrequencies. The actual force also depends on the plate bending rigidity, plate width and the measured displacement. The resulting force is within common linear actuators for typical cases. 


\section{References}

Benjeddou A 2000 Advances in piezoelectric finite element modeling of adaptive structural elements: A survey. Computers and Structures 76: 347-363

Bingham B, Atalla M J, Hagood N W 2001 Comparison of structural-acoustic control designs on an active composite panel. J. Sound and Vibration 244(5): 761-778

Bolotin V V, Grishko A A, Roberts J B, Kounadis A N, Gantes C H 2001 The fluttering panel as a continuous nonlinear nonconservative system. J. Vibration and Control 7: 233-247

Bos F, Casagrande S B 2003 On-line non-destructive evaluation and control of wood-based panels by vibration analysis. J. Sound and Vibration 268: 403-412

Cai G P, Yang S X 2006 A discrete optimal control method for a flexible cantilever beam with time delay. J. Vibration and Control 12(5): 509-526

Chandiramani N K, Librescu L I, Saxena V, Kumar A 2004 Optimal vibration control of a rotating composite beam with distributed piezoelectric sensing and actuation. Smart Materials and Struct. 13(2): 433-442

Chow P L, Maestrello L 1997 Active control of non-linear panel vibration and sound radiation. J. Sound and Vibration 201(3): 390-403

De Fonseca P, Sas P, Van Brussel H 1999 A comparative study of methods for optimizing sensor and actuation locations in active control. J. Sound and Vibration 4: 651-679

De Matteis G, Formisano A, Panico S, Mazzolani F M 2008 Numerical and experimental analysis of pure aluminium shear panels with welded stiffeners. Computers and Struct. 86: 545-555

Döngi F, Dinkler D, Kröplin B 1996 Active panel flutter suppression using self-sensing piezoactuators. AIAA Journal 34(6): 1224-1230

Forster E E, Yang H T Y 1998 Flutter control of wing boxes using piezoelectric actuators. J. Aircraft 35(6): 949-955

Frampton K D, Clark R L, Dowell E H 1996 Active control of panel flutter with piezoelectric transducers. J. Aircraft 33(4): 768-774

Gaudenzi P, Carbonaro R, Barboni R 1997 Vibration control of an active laminated beam. Composite and Struct. 38(1-4): 413-420

Halim D, Barrault G, Cazzolato B S 2008 Active control experiments on a panel structure using a spatially weighted objective method with multiple sensors. J. Sound and Vibration 315: 1-21

Han J H, Tani J, Qui J 2006 Active flutter suppression of a lifting surface using piezoelectric actuation and modern control theory. J. Sound and Vibration 291: 706-722

Han J H, Lee I 1999 Optimal placement of piezoelectric sensors and actuators for vibration control of a composite plate using genetic algorithms. Smart Materials and Struct. 8: 257-267

Hurlebaus S, Stöneber U, Gaul L 2008 Vibration reduction of curved panels by active modal control. Computers and Struct. 86: 251-257

Kwak M K, Heo S 2007 Active vibration control of a smart grid structure by multiinout and multioutput positive position feedback controller. J. Sound and Vibration 304: 230-245

Luo Y, Xie S, Zhang X 2008 Vibration control of honeycomb sandwich panel using multi-layer piezoelectric actuator. Computers and Struct. 86: 744-757

Luo Y, Xie S, Zhang X 2008 The actuated performance of multi-layer piezoelectric actuator in active vibration control of honeycomb sandwich panel. J. Sound and Vibration 317(3-5): 496-513

Moon S H, Kim S J 2005 Feedback linearization control for panel flutter suppression with piezoelectric actuators. AIAA Journal 43(9): 2069-2073

Ro J, Baz A 2002 Vibration control of plates using self-sensing active constrained layer damping networks. J. Vibration and Control 8: 833-845

Sadri A M, Wright J R, Wayne R J 1999 Modelling and optimal placement of piezoelectric actuators in isotropic plates using genetic algorithms. Smart Materials and Struct. 8: 257-267

Shen Y, Homaifar A 2001 Vibration control of flexible structures with PZT sensors and actuators. J. Vibration and Control 7: 417-451 
Shete C D, Chandiramani N K, Librescu L I 2007 Optimal control of pretwisted shearable smart composite rotor blades. Acta Mechanica 191: 37-58

Sunar M, Rao S S 1999 Recent advances in sensing and control of flexible structures via piezoelectric materials technology. Appl. Mech. Rev. 52(1): 1-16

Yang S, Ngoi B 2000 Shape control of beams by piezoelectric actuators. AIAA Journal 38(12): 2292-2298

Varadarajan S, Chandrashekhara K, Agarwal S 1998 Adaptive shape control of laminated composite plates using piezoelectric materials. AIAA Journal 36(9): 1694-1698

Walker L A, Yaneske P P 1976 Characteristics of an active feedback system for the control of plate vibrations. J. Sound and Vibration 46: 157-176

Young T H, Tseng T C, Song L S 2002 Dynamic stability of fluttered systems subjected to parametric random excitations. J. Vibration and Control 8: 291-310 